\title{
Comprehensive Analysis On A New Type VR-Resolver with Toroidal Windings Under Healthy and Eccentric Cases
}

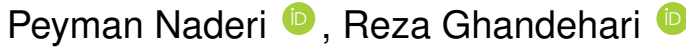

\begin{abstract}
A novel analysis for a new type Variable Reluctance Resolver (VR-Resolver) with toroidal windings is presented in this work. The resolver with various windings configurations as well as different rotor structures is considered for analysis. Position error of the resolvers in both healthy and eccentric cases are studied, where the effect of the eccentricity fault is analyzed for the resolvers with various windings configuration and rotor shapes. Hence, the analysis of the manufacturing tolerance due to the eccentric rotor is obtained by several analyses. Noticing the Magnetic Equivalent Circuit (MEC) method's flexibility as well as in regards to the analysis of the several structures, a well-known flexible MEC-based method with adjustable accuracy is considered for modeling. Hence shorter processing time and more flexibility compared to Finite-ElementMethod (FEM) are needed. Finally, the effectiveness of the presented works is proved by the performed validation via both FEM and experimental results.
\end{abstract}

Index Terms-Eccentricity fault, Magnetic Equivalent Circuit, toroidal windings, variable reluctance resolver.

\section{INTRODUCTION}

$\mathbf{V}$ ARIABLE reluctance resolvers (VR-Resolver) is an accurate position sensor that is necessary in many industries such as inverter-driven electric machines, robotic, and vehicular technologies [1]. Although optical encoders are wellknown position sensors, they have important disadvantages such as working in environments with high temperature, smoke, and dust. Moreover, the encoders have speed limitation, where big position error is obtained in high-speed applications [2]. Resolvers are generally categorized into two types. The first type is Wound Rotor Resolver (WR-Resolver) [3]-[7] that have a connected voltage scourge by slip rings, and a rotary transformer is necessary [6]. In [6], its accuracy improvement by the arrangement of the windings is addressed, and in [7] the accuracy is analyzed under different installation

Manuscript received Month $\mathrm{xx}, 2 \mathrm{xxx}$; revised Month $\mathrm{xx}, \mathrm{xxxx}$; accepted Month $\mathrm{x}, \mathrm{xxxx}$. This work was supported in part by the $\mathrm{xxx}$ Department of xxx under Grant (sponsor and financial support acknowledgment goes here).

Peyman Naderi is associate professor in Electrical Engineering Department, Shahid Rajaee Teacher Training University, Tehran, 1678815811, Iran. (e-mail: p.naderi@sru.ac.ir).

Reza Ghandehari is associate professor in Electrical Engineering Department, Shahid Rajaee Teacher Training University, Tehran, 1678815811, Iran. (e-mail: a_ghandehari@sru.ac.ir). configurations. Although in [5] eliminating the core of the rotary transformer a new type WR-Resolver is presented, that is more costly [8] compared to the second type which is Variable Reluctance Resolver (VR-Resolver). In this type, the rotor is a solid magnetic material with $m$ number of rotor saliency (called $\mathrm{m}-\mathrm{X}$ VR-Resolver), where the windings are located on the stationary part. The rotor shape of the VR-Resolvers is considered based on the sinusoidal permeance [9], [10]. Hence various rotor shapes [9], [11] and its optimization [2], [10], [11] are investigated in some researches. Noticing the VRResolvers benefit, there are some new researches which have focused on its development. Hall Effect Sensor (HS) usage in the VR-Resolvers is one of the new investigated techniques which is presented in [12]-[14]. In [12], [13] both excitation winding and PMs are used, where Back-Emf enhancement is performed by PMs and various VR-Resolvers are presented. In [14] Permanent Magnets (PMs) are used as the excitation and the position calculation is based on the measured fluxes by the HSs. Although the absence of the high-frequency excitation signal is known as an advantage, the HSs' sensitivity to the external magnetic fields and environment temperature are its disadvantages. The axial flux VR-Resolvers are another new type of resolvers that are investigated by researchers. In [15] the axial flux VR-Resolver is addressed, where a fast computational method is presented for its modeling. A novel VR-Resolver with toroidal windings is analyzed in [16], where two windings configurations are analyzed and the manufacturing tolerance of the resolver with both configurations is studied. Moreover, windings arrangement simplification is presented in [17] and position error analysis is done under the simplified case. Noticing the performed researches, there are three considered methods for the resolvers modeling. The Finite Element Method (FEM) is a powerful method that has been used many times but has some disadvantages such as long processing time and intensive processing. In [18], challenges of the FEM for resolvers modeling is illustrated and necessary considerations are analyzed. Magnetic Equivalent Circuit (MEC) is another choice that is more flexible compared to FEM, where less computational time can be obtained by that. This method is used recently for resolvers modeling thanks to its capability, where the WR and VR types are analyzed by that in [5] and [15], [19]-[21], respectively. Moreover, PM-Resolver model by MEC is addressed in [14], and the VR-Resolver with toroidal windings is modeled by 
that in [16]. In the presented works, the linear core is considered and the model accuracy is not adjustable. Although the resolvers operating point is low in the normal case, that may be near to the knee-point of the B-H curve in intensive eccentricity fault or with a low-frequency excitation in lowspeed applications. Hence, the saturation effect is suitable for an integrated model of the resolvers.

As is illustrated previously, the VR-Resolver with toroidal windings is a new type of resolver which is presented firstly in [16]. In the presented work, two types of windings configurations are analyzed and the manufacturing tolerance is investigated for the resolver with 5 rotor saliencies $(5-\mathrm{X}$ VR-Resolver) by a simple MEC technique. This paper is an extended version of the mentioned work, where its novelties are summarized as follows:

- A flexible MEC-based method with adjustable accuracy is used for modeling.

- In addition to the presented windings configurations in [16], an additional configuration is analyzed.
- The position error under four different healthy rotor types are obtained.

- The position error under healthy and eccentric rotors are analyzed, where different windings configurations, as well as different rotor shapes, are considered.

Notably, since the static eccentricity has more impact on the resolvers' performance compared to dynamic type [20], [21], the static eccentricity is considered for the analysis.

\section{AnAlyzed Resolvers}

The investigated resolvers are presented in Fig. 1, where the considered windings configurations and the rotor shapes are shown. The windings direction and its number of turners per slot are illustrated in Table I, where Wsin, Wcos, and Wext denotes sine, cosine, and excitation windings, as well as $N_{s}$ and $N_{e}$, are the maximum number of turn per toroidal coil of the signals and excitation windings, respectively.

TABLE I

CONSIDERED WINDINGS CONFIGURATION

\begin{tabular}{|c||c|c|c|c|c|c|c|c|c|c|c|c|}
\hline WT1 & S1 & S2 & S3 & S4 & S5 & S6 & S7 & S8 & S9 & S10 & S11 & S12 \\
\hline Wsin & 0 & $N_{s} \uparrow$ & 0 & 0 & 0 & $N_{s} \downarrow$ & 0 & $N_{s} \downarrow$ & 0 & 0 & 0 & $N_{s} \uparrow$ \\
\hline Wcos & 0 & 0 & $N_{s} \uparrow$ & 0 & $N_{s} \uparrow$ & 0 & 0 & 0 & $N_{s} \downarrow$ & 0 & $N_{s} \downarrow$ & 0 \\
\hline Wext & $N_{e} \uparrow$ & 0 & 0 & $N_{e} \downarrow$ & 0 & 0 & $N_{e} \uparrow$ & 0 & 0 & $N_{e} \downarrow$ & 0 & 0 \\
\hline \hline WT2 & $\mathbf{S 1}$ & $\mathbf{S 2}$ & $\mathbf{S 3}$ & $\mathbf{S 4}$ & $\mathbf{S 5}$ & $\mathbf{S 6}$ & $\mathbf{S 7}$ & $\mathbf{S 8}$ & $\mathbf{S 9}$ & $\mathbf{S 1 0}$ & $\mathbf{S 1 1}$ & $\mathbf{S 1 2}$ \\
\hline Wsin & $N_{s} \uparrow$ & $N_{s} \downarrow$ & 0 & 0 & $N_{s} \downarrow$ & $N_{s} \uparrow$ & $N_{s} \downarrow$ & $N_{s} \uparrow$ & 0 & 0 & $N_{s} \uparrow$ & $N_{s} \downarrow$ \\
\hline Wcos & 0 & $N_{s} \downarrow$ & $N_{s} \uparrow$ & $N_{s} \downarrow$ & $N_{s} \uparrow$ & 0 & 0 & $N_{s} \uparrow$ & $N_{s} \downarrow$ & $N_{s} \uparrow$ & $N_{s} \downarrow$ & 0 \\
\hline Wext & $N_{e} \uparrow$ & 0 & $N_{e} \downarrow$ & $N_{e} \uparrow$ & 0 & $N_{e} \downarrow$ & $N_{e} \uparrow$ & 0 & $N_{e} \downarrow$ & $N_{e} \uparrow$ & 0 & $N_{e} \downarrow$ \\
\hline \hline WT3 & $\mathbf{S 1}$ & $\mathbf{S 2}$ & $\mathbf{S 3}$ & $\mathbf{S 4}$ & $\mathbf{S 5}$ & $\mathbf{S 6}$ & $\mathbf{S 7}$ & $\mathbf{S 8}$ & $\mathbf{S 9}$ & $\mathbf{S 1 0}$ & $\mathbf{S 1 1}$ & $\mathbf{S 1 2}$ \\
\hline Wsin & 0 & $0.5 N_{s} \uparrow$ & $0.87 N_{s} \uparrow$ & $N_{s} \uparrow$ & $0.87 N_{s} \uparrow$ & $0.5 N_{s} \uparrow$ & 0 & $0.5 N_{s} \downarrow$ & $0.87 N_{s} \downarrow$ & $N_{s} \downarrow$ & $0.87 N_{s} \downarrow$ & $0.5 N_{s} \downarrow$ \\
\hline Wcos & $N_{s} \uparrow$ & $0.87 N_{s} \uparrow$ & $0.5 N_{s} \uparrow$ & 0 & $0.5 N_{s} \downarrow$ & $0.87 N_{s} \downarrow$ & $N_{s} \downarrow$ & $0.87 N_{s} \downarrow$ & $0.5 N_{s} \downarrow$ & 0 & $0.5 N_{s} \uparrow$ & $0.87 N_{s} \uparrow$ \\
\hline Wext & $N_{e} \uparrow$ & $N_{e} \downarrow$ & $N_{e} \uparrow$ & $N_{e} \downarrow$ & $N_{e} \uparrow$ & $N_{e} \downarrow$ & $N_{e} \uparrow$ & $N_{e} \downarrow$ & $N_{e} \uparrow$ & $N_{e} \downarrow$ & $N_{e} \uparrow$ & $N_{e} \downarrow$ \\
\hline
\end{tabular}
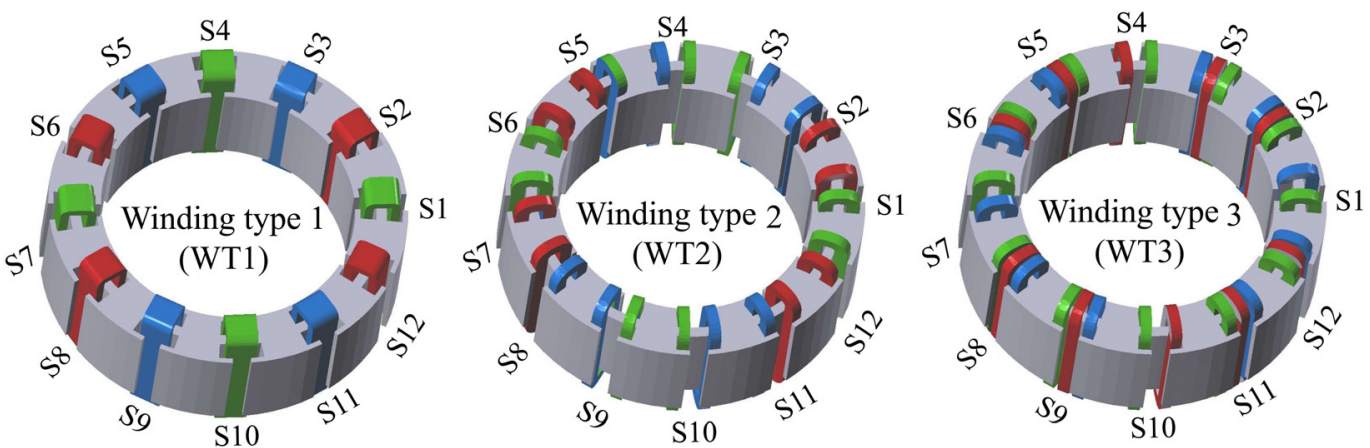

Green, Blue, and Red windings denotes Excitation (Wext), Cosine (Wcos) and Sine (Wsin) windings
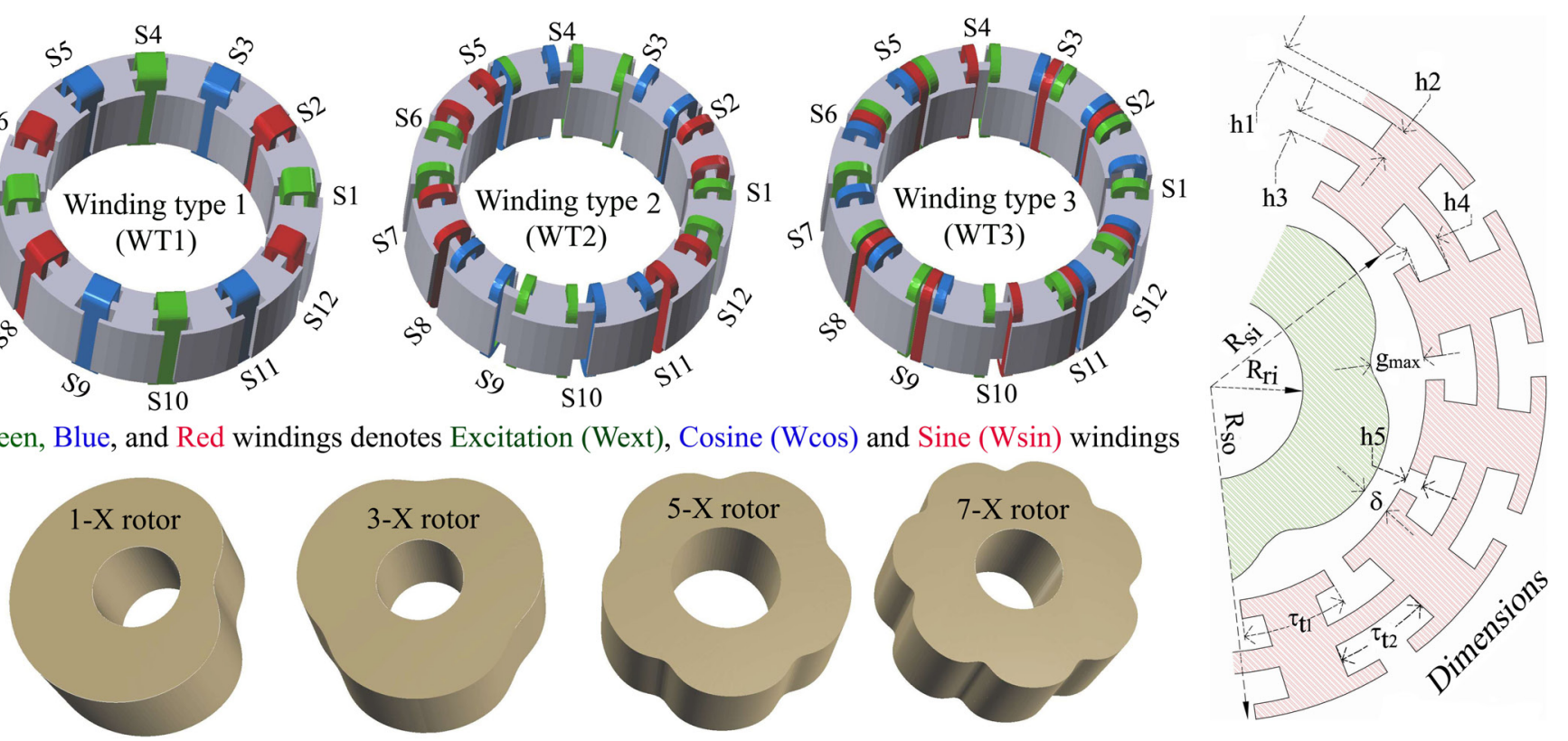

Fig. 1. Investigated resolvers with dimensions, various windings configurations, and rotor types, where Si denotes $\mathrm{i}^{\text {th }}$ slot 


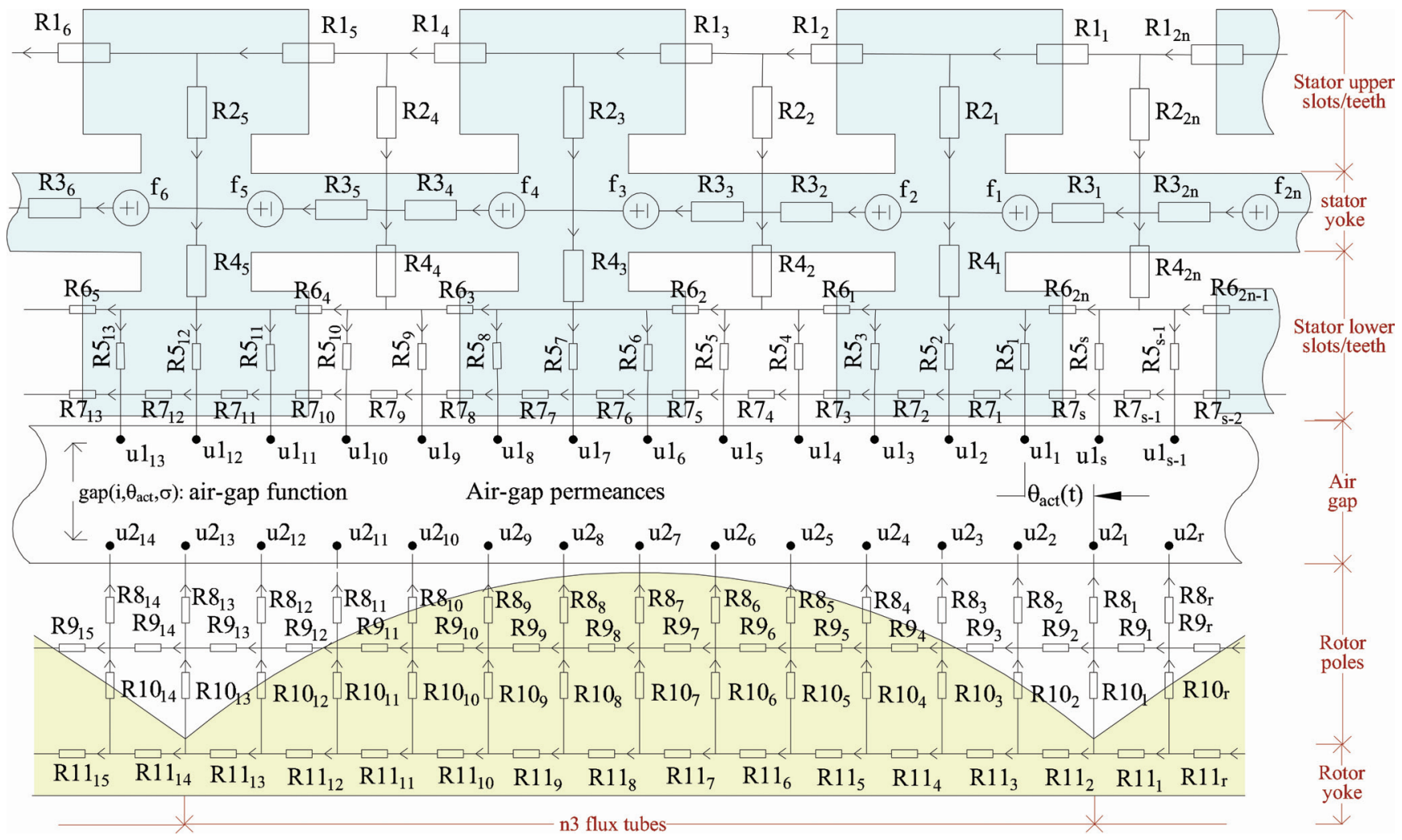

Fig. 2. Considered MEC model with a sample accuracy $\left(n_{f 1}=3, n_{f 2}=2, n_{f 3}=12\right)$

TABLE II

Naming of the Analyzed Resolvers With Toroidal Windings

\begin{tabular}{c|c|c|c|c|}
\cline { 3 - 5 } \multicolumn{2}{c|}{} & \multicolumn{3}{c|}{ Windings Configuration (Stator) } \\
\cline { 2 - 5 } \multicolumn{2}{c|}{} & WT1 & WT2 & WT3 \\
\hline \multirow{3}{*}{$心$} & $1-\mathrm{X}$ & W1R1 & W2R1 & W2R1 \\
\cline { 2 - 5 } & $3-\mathrm{X}$ & $\mathrm{W} 1 \mathrm{R} 3$ & W2R3 & W3R3 \\
\cline { 2 - 5 } & $5-\mathrm{X}$ & $\mathrm{W} 1 \mathrm{R} 5$ & W2R5 & W2R5 \\
\cline { 2 - 5 } & $7-\mathrm{X}$ & $\mathrm{W} 1 \mathrm{R} 7$ & $\mathrm{~W} 2 \mathrm{R} 7$ & W3R7 \\
\hline
\end{tabular}

It is notable that just two first windings configurations (WT1 and WT2) with a 5-X rotor are analyzed in [16] for healthy case, where all the structures are analyzed in this paper under both healthy and eccentric cases in this paper. In regards to Fig. 1, there are 12 possible structures for the analyzed VRResolvers, where their naming is introduced in Table II.

\section{MEC-Based Model of the Resolver}

Since there are 12 cases for analysis, an 11-zone MECbased technique is considered for simulations thanks to its flexibility and shorter processing time compared to FEM.

\section{A. Considered MEC}

The considered MEC-based model is flexible to the model of all rotor/stator structures with adjustable accuracy, where the number of flux tubes (FLs) in the sensitive zones can be adjusted by the user. The resolver structure and its accuracy can be adjusted by the number of FLs as written in Table III, where a sample accuracy $\left(n_{f 1}=3, n_{f 2}=2\right.$, and $\left.n_{f 3}=12\right)$ is shown in Fig. 2. The $R i_{j}$ and $\phi i_{j}$ denotes reluctance and flux of $j^{\text {th }} \mathrm{FL}$ in $i^{\text {th }}$ zone, respectively. Each FL is modeled by Eq. (1), where $l i_{j}^{c}$ and $l i_{j}^{g}$ denotes length of the core and air parts of the $j^{\text {th }} \mathrm{FL}$ in $i^{\text {th }}$ zone, respectively.
TABLE III

Selective Parameters of MEC Model for VR-Resolver

\begin{tabular}{|c|l|}
\hline \multicolumn{2}{|c|}{ Properties of $\mathbf{~ m - X}$ VR-Resolver } \\
\hline Symbol & Definition \\
\hline$n$ & Number of stator teeth \\
& Number of rotor poles (called $m-\mathrm{X}$ resolver) \\
\hline \multicolumn{2}{|c|}{ Number of flux tubes per each part } \\
\hline$n_{f 1}$ & Definition \\
$n_{f 2}$ & Number of flux tubes per mover tooth \\
$n_{f 3}$ & Number of flux tubes per mover slot \\
\hline \multicolumn{2}{|c|}{ Total number of flux tubes per each zone } \\
\hline Zone number & Number of flux tubes in the zone \\
\hline $1,2,3,4,6$ & $2 n$ \\
5,7 & $s=n \times\left(n_{f 1}+n_{f 2}\right)$ \\
$8,9,10,11$ & $r=m \times n_{f 3}$ \\
\hline
\end{tabular}

$$
R i_{j}=\frac{l i_{j}^{g}}{\mu_{0} A i_{j}}+\frac{l i_{j}^{c}}{\mu_{0} \mu_{r}\left(B i_{j}\right) A i_{j}}
$$

In Eq. (1), $B i_{j}$ and $A i_{j}$ are flux density and effective area of the FL, respectively. Moreover, $\mu_{r}\left(B i_{j}\right)$ is defined as a functional relative permeability of the FL to model of the $\mathrm{B}-\mathrm{H}$ curve nonlinearity. It is a keynote that normally the resolvers operating points are low thanks to high-frequency excitation, but that may be investigatable in some cases such as intensive eccentricity. Additionally, for low-speed applications, low-frequency excitation is possible. Hence, the operation point may be considerable in low-frequency excitation which is possible for low-speed applications. Therefore, modeling the saturation effect is necessary for an integrated model. 


$$
\begin{aligned}
& \mathbf{A}(\mathbf{X}(t)) \mathbf{X}(t)=\mathbf{B}(V e(t), V e(t-\Delta t), \mathbf{X}(t-\Delta t)) \rightarrow \mathbf{X}(t)=\mathbf{A}(\mathbf{X}(t))^{-1} \mathbf{B}(V e(t), V e(t-\Delta t), \mathbf{X}(t-\Delta t))
\end{aligned}
$$

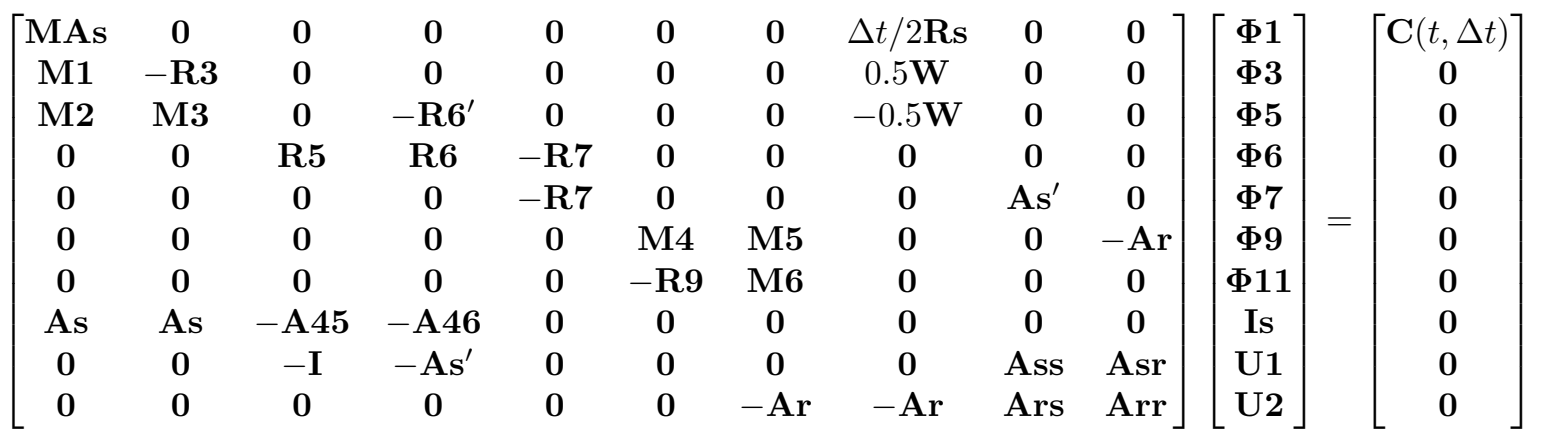

There are some well-known equations which are used recently for relative permeability function. The below equation is used in this paper for that which allows having saturation and core nonlinearity effects [20], [21].

$$
\mu_{r}\left(B i_{j}\right)=\frac{a}{b B i_{j}^{4}-c B i_{j}^{2}+d}
$$

\section{B. MEC-Based Equations}

Considering $V_{e}(t)$ as the excitation windings voltage, the $\mathbf{W}$ and $\mathbf{M}$ should be obtained based on the windings configuration. Moreover, by using $\Delta t$ as the time-step, the whole equations can be written as Eq. (2). Details of the equations and solving procedure are complectly illustrated in [20], [21]. Noticing Fig. 2 the $\mathbf{W}$ and $\mathbf{M}(2 n \times 3$ and $3 \times 2 n$ matrices) in Eq. (2) should be computed as below, where $N_{a i}, N_{b i}$, and $N_{c i}$ are the number of turns in the $\mathrm{Si}^{\text {th }}$ slot for Wsin, Wcos, and Wext, windings, respectively.

$$
\mathbf{W}=\left[\begin{array}{ccc}
N_{a 2} & N_{b 2} & N_{c 2} \\
N_{a 2} & N_{b 2} & N_{c 2} \\
N_{a 3} & N_{b 3} & N_{c 3} \\
N_{a 3} & N_{b 3} & N_{c 3} \\
\vdots & \vdots & \vdots \\
N_{a n} & N_{b n} & N_{c n} \\
N_{a n} & N_{b n} & N_{c n} \\
N_{a 1} & N_{b 1} & N_{c 1} \\
N_{a 1} & N_{b 1} & N_{c 1}
\end{array}\right], \mathbf{M}^{T}=\left[\begin{array}{ccc}
N_{a 1} & N_{b 1} & N_{c 1} \\
N_{a 2} & N_{b 2} & N_{c 2} \\
N_{a 2} & N_{b 2} & N_{c 2} \\
N_{a 3} & N_{b 3} & N_{c 3} \\
N_{a 3} & N_{b 3} & N_{c 3} \\
\vdots & \vdots & \vdots \\
N_{a n} & N_{b n} & N_{c n} \\
N_{a n} & N_{b n} & N_{c n} \\
N_{a 1} & N_{b 1} & N_{c 1}
\end{array}\right]
$$

It is a keynote that the healthy and eccentric resolvers can be modeled by the considered MEC in Fig. 2. In [20], [21], the modeling technique for healthy and eccentric rotors are presented, where the calculation of the defined air-gap permeance in Eq. (5) based on the air-gap function $\operatorname{gap}\left(\theta_{\text {act }}, i, \sigma_{d}, \sigma_{s}\right)$ are addressed in details. In Eq. (5), $0 \leqslant \sigma_{d} \leqslant 1$ and $0 \leqslant \sigma_{s} \leqslant 1$ denotes dynamic and static eccentricity indexes.

$$
G_{m}\left(\theta_{\text {act }}, i, \sigma_{s}, \sigma_{d}\right)=\mu_{0} \times l \times \frac{\min \left(\gamma_{s} R_{s i}, \gamma_{r} R_{r o}\right)}{\operatorname{gap}\left(\theta_{\text {act }}, i, \sigma_{s}, \sigma_{d}\right)}
$$

Moreover, as illustrated in [20], [21], the rotor shapes are considered by Eq. (6) to produce sinusoidal air-gap permeance.

$$
l 8_{j}^{g}+l 10_{j}^{g}=\frac{1}{\alpha_{1}-\alpha_{2} \cos \left(m(j-1) \gamma_{r}\right)}-\delta=f g(j)
$$

In Eq. (6) $\alpha_{1}, \alpha_{2}$ and $\delta$ are defined for minimum and maximum air-gap lengths, and $\gamma_{s}$, and $\gamma_{r}$ are FLs pitch as shown in Eq. (7).

$$
\left\{\begin{array}{l}
\frac{1}{\alpha_{1}+\alpha_{2}}=\delta, \frac{1}{\alpha_{1}-\alpha_{2}}=g_{\max } \\
\gamma_{s}=\frac{2 \pi}{s}, \gamma_{r}=\frac{2 \pi}{r}
\end{array}\right.
$$

\section{Simulation AND ANALYSIS}

Simulations are performed for the resolver with considered properties in Table IV, where the resolver dimensions are shown in Fig. 1. In all simulations, $1000 \mathrm{rpm}$ is considered as the rotor speed as well as a $4.2 \mathrm{~V}_{\mathrm{rms}}, 5 \mathrm{kHz}$ voltage is applied to the excitation winding.

Simulations are performed for all defined resolvers in Table II,

\begin{tabular}{|c|c|c|c|c|c|}
\hline \multicolumn{6}{|c|}{ Electrical and Structural Properties } \\
\hline \multirow{2}{*}{ Parameter } & \multirow{2}{*}{ Symb. } & \multicolumn{4}{|c|}{ Value } \\
\hline & & R1 & R3 & R5 & R7 \\
\hline Number of stator teeth/slots & $n$ & \multicolumn{4}{|c|}{12} \\
\hline Number of rotor poles & $m$ & 1 & 3 & 5 & 7 \\
\hline Number of turns in signal winding & $N_{s}$ & \multicolumn{4}{|c|}{20} \\
\hline Number of turns in excitation winding & $N_{e}$ & \multicolumn{4}{|c|}{20} \\
\hline Resistance of excitation winding $(\Omega)$ & $R_{e}$ & \multicolumn{4}{|c|}{2} \\
\hline \multicolumn{6}{|c|}{ Dimensions } \\
\hline Arc of stator teeth (Deg.) & $\tau_{t 1}$ & \multicolumn{4}{|c|}{18} \\
\hline Arc of stator slots (Deg.) & $\tau_{t 2}$ & \multicolumn{4}{|c|}{20} \\
\hline Height of stator teeth tips (mm) & $h 1, h 5$ & \multicolumn{4}{|c|}{1} \\
\hline Height of stator slots $(\mathrm{mm})$ & $h 2, h 4$ & \multicolumn{4}{|c|}{2.5} \\
\hline Height of stator yoke (mm) & h3 & \multicolumn{4}{|c|}{1} \\
\hline Minimum length of air-gap (mm) & $\delta$ & \multicolumn{4}{|c|}{0.5} \\
\hline Maximum length of air-gap (mm) & $\mathrm{g}_{\max }$ & 5 & & 2 & 2 \\
\hline stator outer radius $(\mathrm{mm})$ & $R_{\text {so }}$ & \multicolumn{4}{|c|}{20} \\
\hline Stator inner radius $(\mathrm{mm})$ & $R_{s i}$ & \multicolumn{4}{|c|}{12} \\
\hline Rotor outer radius $(\mathrm{mm})$ & $R_{\text {ro }}$ & \multicolumn{4}{|c|}{11.5} \\
\hline \multicolumn{6}{|c|}{ Considered Accuracy in the MEC Model } \\
\hline Number of FLs per stator tooth & $n_{f 1}$ & \multicolumn{4}{|c|}{6} \\
\hline Number of FLs per stator slot & $n_{f 2}$ & \multicolumn{4}{|c|}{4} \\
\hline Number of FLs per rotor poles & $n_{f 3}$ & 180 & 60 & 32 & 22 \\
\hline
\end{tabular}
where the bellow scenarios are considered:

A)-Healthy resolvers.

B)-Static eccentric resolvers under $\sigma_{s}=0.5$.

TABLE IV

Parameters of Simulated $m-X$ TOROIDAL VR-RESOlVERS 
The bellow considerations are used to obtain the results:

Since the Resolver to Digital (R/D) converter is not considered in this paper, in all simulations, the Hilbert transformation is used to obtain the envelope signals, where a written m-file in the Matlab software is used for positive/negative half-cycles [21]. In each case, the envelope functions ( $V_{\text {sine }}$ and $\left.V_{\text {cosine }}\right)$ are obtained from the induced voltages in the signal windings $\left(V_{\mathrm{Wsin}}\right.$ and $\left.V_{\mathrm{Wcos}}\right)$. Then, the calculated position and the position error are obtained by Eq. (8) for a $m-\mathrm{X}$ resolver.

$$
\left\{\begin{array}{l}
\theta_{\text {calulate }}(t)=\frac{1}{m} \tan ^{-1}\left(\frac{V_{\text {sine }}(t)}{V_{\text {cosine }}(t)}\right) \\
\theta_{\text {error }}(t)=\theta_{\text {actual }}(t)-\theta_{\text {calculate }}(t)
\end{array}\right.
$$

W Considering $1000 \mathrm{rpm}$ as the rotational speed, $60 \mathrm{~ms}$ duration time is necessary for a $360^{\circ}$ rotation. In order to avoid the transient performance, $65 \mathrm{~ms}$ simulation is performed, where the results for $2.5^{\mathrm{ms}} \leqslant t \leqslant 62.5^{\mathrm{ms}}$ are analyzed. The actual initial position is tuned manually as $\theta_{\text {actual }}(0)=-15^{\circ}$. Hence $\theta_{\text {actual }}\left(2.5^{\mathrm{ms}}\right)=0^{\circ}$ is obtained for absolute position error analysis.

Considering $5 \mathrm{kHz}$ excitation frequency and $1000 \mathrm{rpm}$ rotational speed, $\Delta t=1.25 \mu \mathrm{s}$ is used as the time steep in the MEC method (see Eq. (2b)) to leads to trustable results. Noticing Eq. (8), the zero position is calculated by $V_{\text {sine }}(t)=0$ which should be obtained at $\theta_{\text {actual }}(2 k \pi)$ for $k=0,1, \ldots$. Clearly, the mentioned values can be obtained by an ideal sensor. Hence, the calculated value by a resolver is considered as the absolute position error of the resolver.

\section{A. Healthy Resolvers}

Performance of the healthy resolvers is evaluated in this part. Since there are 24 analyzed cases, details of some cases are shown in Figs. 3-5. The induced voltages, envelope functions, and position errors are shown in the figures for W1R1, W1R5, and W3R5 cases. The maximum and absolute position errors, as well as peak of the induced voltages of the healthy resolvers, are listed in Table V under $1000 \mathrm{rpm}$, where $60 \mathrm{~ms}$ duration time denotes a $360^{\circ}$ rotation.

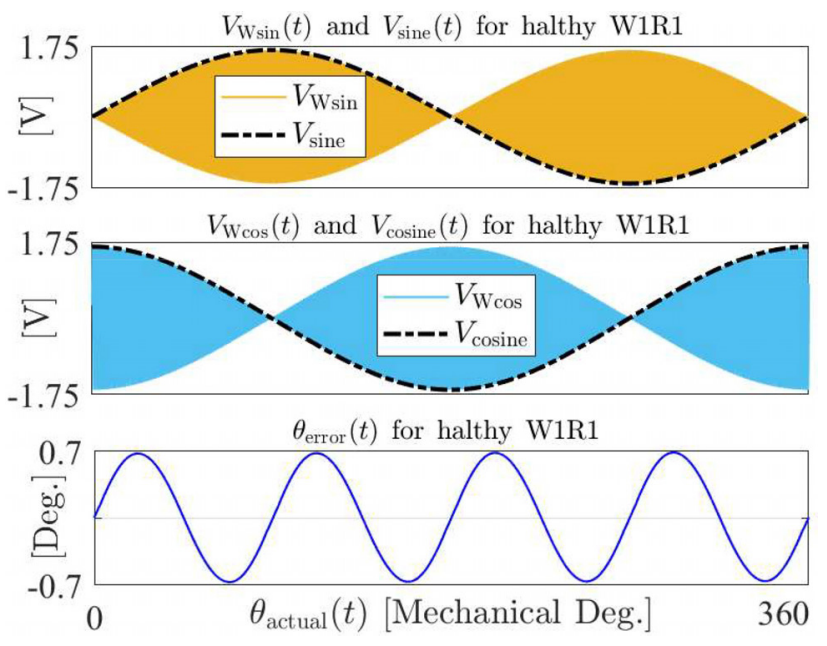

Fig. 3. Induced voltages and envelopes functions for healthy W1R1

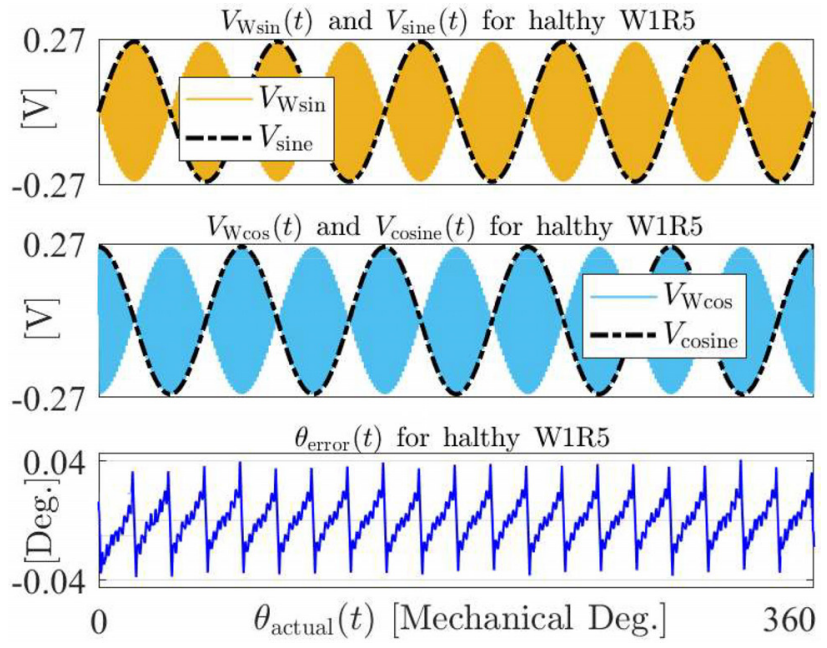

Fig. 4. Induced voltages and envelopes functions for healthy W1R5
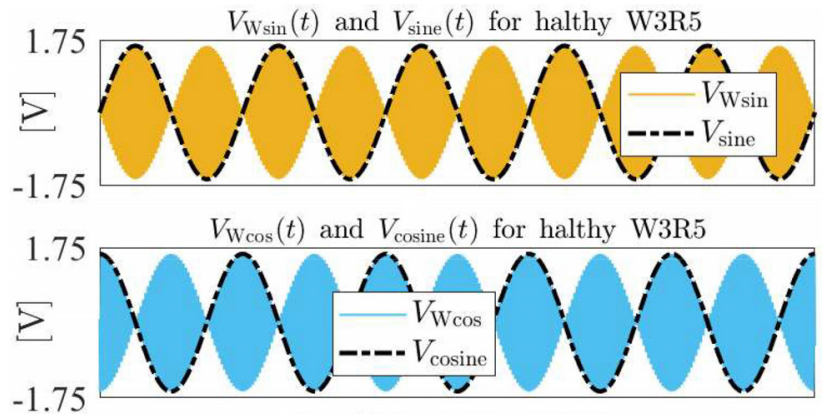

$-1.75$

$\theta_{\text {error }}(t)$ for halthy W3R5

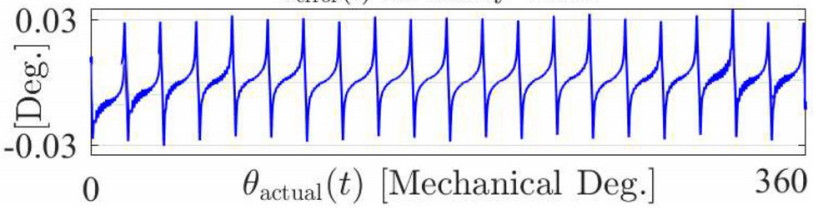

Fig. 5. Induced voltages and envelopes functions for healthy W3R5

TABLE V

Results of the Analysis on the Healthy Resolvers

\begin{tabular}{|c|c|c|c|}
\hline \multicolumn{4}{|c|}{ Maximum position error (Deg.) } \\
\hline W1R1 & W1R3 & W1R5 & W1R7 \\
\hline 0.7 & 0.04 & 0.04 & 0.20 \\
\hline W2R1 & W2R3 & W2R5 & W2R7 \\
\hline 0.47 & Not applicable & 0.084 & 0.037 \\
\hline W3R1 & W3R3 & W3R5 & W3R7 \\
\hline Not applicable & Not applicable & 0.03 & 0.025 \\
\hline \multicolumn{4}{|c|}{ Absolute position error (Deg.) } \\
\hline W1R1 & W1R3 & W1R5 & W1R7 \\
\hline 0.0057 & 0.009 & 0.0098 & 0.00095 \\
\hline W2R1 & W2R3 & W2R5 & W2R7 \\
\hline-0.114 & - & 0.009 & 0.0002 \\
\hline W3R1 & W3R3 & W3R5 & W3R7 \\
\hline- & - & 0.0099 & 0.0075 \\
\hline \multicolumn{4}{|c|}{ Peak of the induced voltages $(\boldsymbol{V})$} \\
\hline W1R1 & W1R3 & W1R5 & W1R7 \\
\hline 1.75 & 1.8 & 0.27 & 0.195 \\
\hline W2R1 & W2R3 & W2R5 & W2R7 \\
\hline 0.67 & - & 0.47 & 0.32 \\
\hline W3R1 & W3R3 & W3R5 & W3R7 \\
\hline- & - & 1.75 & 1.33 \\
\hline
\end{tabular}


Noticing the obtained values, there are three non-applicable cases (W2R3, W3R1, and W3R3) in which non-sinusoidal induced voltages are produced. Moreover, the peak of the induced in the W1R5, W1R7, and W2R7 is low compared to other cases. Hence their signal-to-noise ratio is more considerable for them compared to other cases.

\section{B. Eccentric Resolver}

Eccentric resolvers performances are evaluated in this part, where the detail of some cases are shown in Figs. 6-8 as three samples. Noticing the figures, the asymmetry induced voltages ( $V_{\mathrm{Wsin}}$ and $\left.V_{\mathrm{Wcos}}\right)$ is produced due to eccentricity which caused to asymmetry envelope functions $\left(V_{\text {sine }}\right.$ and $\left.V_{\text {cosine }}\right)$. In regards to details of the sample results, the W1R5 and W1R7 resolvers have a very high-level sensitivity, where its position error reaches to infinite value in the eccentric case, while the W3R5 resolver has a low-level sensitivity. Now, the resolvers' sensitivity to the eccentric rotor (also manufacturing tolerance) can be analyzed. Results of the maximum and absolute position errors are tabulated in Table VI.
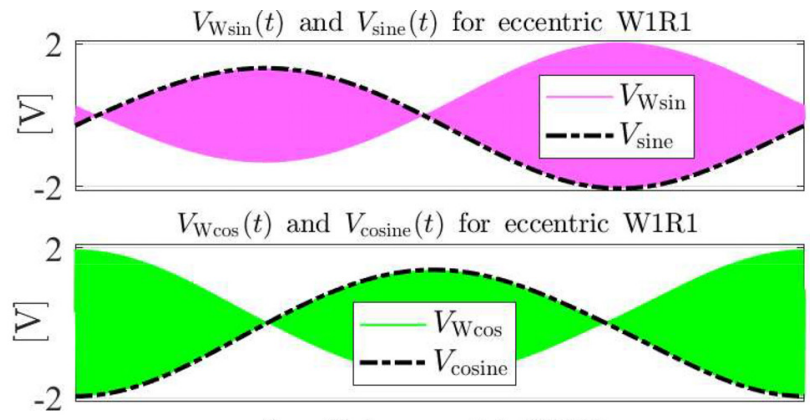

$\theta_{\text {error }}(t)$ for eccentric W1R1

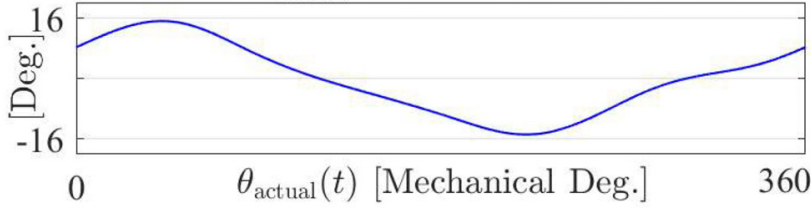

Fig. 6. Induced voltages and envelopes functions for eccentric W1R1
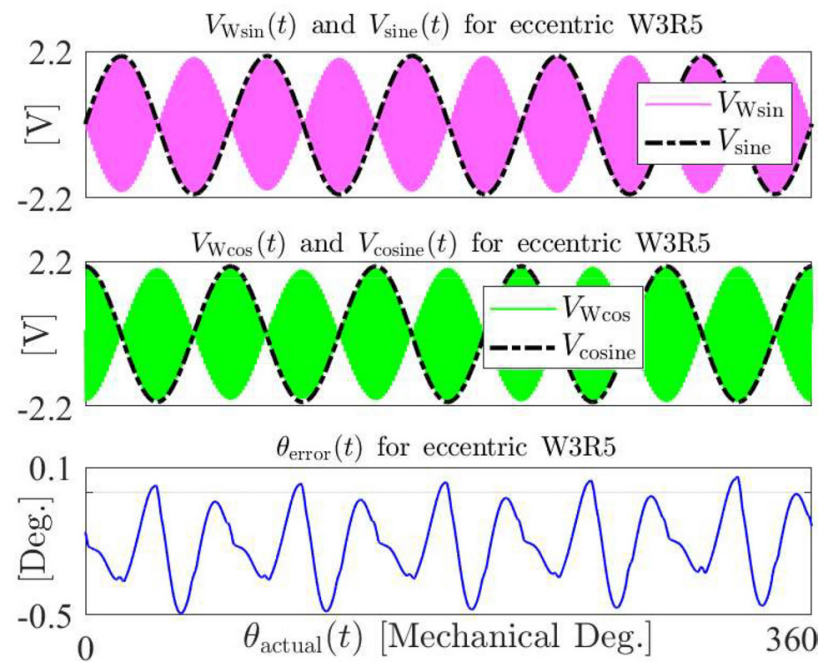

Fig. 7. Induced voltages and envelopes functions for eccentric W3R5

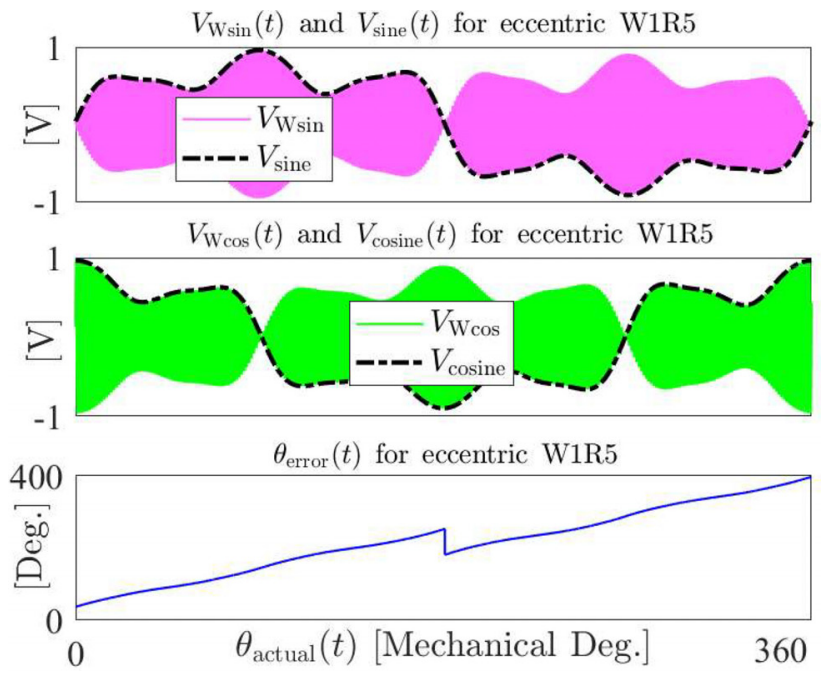

Fig. 8. Induced voltages and envelopes functions for eccentric W1R5

TABLE VI

Maximum and Absolute ErRoRs for the Eccentric Resolvers

\begin{tabular}{|c|c|c|c|}
\hline \multicolumn{5}{|c|}{ Maximum position error (Deg.) } \\
\hline W1R1 & W1R3 & W1R5 & W1R7 \\
\hline 15.2 & 7.80 & $\infty$ & $\infty$ \\
\hline W2R1 & W2R3 & W2R5 & W2R7 \\
\hline 26 & - & 9.62 & 36.32 \\
\hline W3R1 & W3R3 & W3R5 & W3R7 \\
\hline- & -- & 0.50 & 0.46 \\
\hline \multicolumn{4}{|c|}{ Absolute position error (Deg.) } \\
\hline W1R1 & W1R3 & W1R5 & W1R7 \\
\hline 8.32 & 7.58 & $\infty$ & $\infty$ \\
\hline W2R1 & W2R3 & W2R5 & W2R7 \\
\hline 12.6 & - & -10.21 & -9.92 \\
\hline W3R1 & W3R3 & W3R5 & W3R7 \\
\hline- & - & -0.16 & -0.43 \\
\hline
\end{tabular}

\section{Discussion and Resolvers Evaluation}

Regarding the performed analysis, the resolvers' capability and their applications can be discussed. Clearly, manufacturing tolerance is an existing natural phenomenon that should be considered in practical applications. Hence, a low-level eccentricity fault is a considerable fault that may naturally exist in a resolver. In addition, high voltage peak and low position error are valuable factors which should be compared for the analyzed resolvers. Result of the comparison is tabulated in Table VII. As is clear the W5R5 and W5R7 are trustable cases that can be considered as two practical resolvers.

\section{Comparison with 2D-FEM}

In order to validate and compare, the W2R5 resolver in both healthy and faulty cases is simulated by Maxwell software. The resolver geometry, flux density, and flux lines are shown in Fig. 9. Moreover, the induced voltages in both healthy and eccentric cases $\left(\sigma_{s}=0.5\right)$ are demonstrated in Fig. 10 in Maxwell environment. In addition, the obtained position error by both FEM and MEC are compared in Fig. 11 for the eccentric resolver. Noticing the obtained results, there is very good agreement between the results. Hence, the used flexible MEC method capability is confirmed. 
TABLE VII

REsolvers EVALUATION

\begin{tabular}{|c|c|c|c|c|c|c|c|c|c|c|c|c|}
\hline & \multicolumn{4}{|c|}{ WT1 } & \multicolumn{4}{|c|}{ WT2 } & \multicolumn{4}{|c|}{ WT3 } \\
\hline & W1R1 & W1R3 & W1R5 & W1R7 & W2R1 & W2R3 & W2R5 & W2R7 & W3R1 & W3R3 & W3R5 & W3R7 \\
\hline Acceptable position error & No & Yes & Yes & No & No & - & Yes & Yes & - & - & Yes & Yes \\
\hline Acceptable voltage peak & Yes & Yes & No & No & Yes & - & Yes & Yes & - & - & Yes & Yes \\
\hline Acceptable sensitivity to $\mathrm{SE}$ & No & No & No & No & No & No & No & No & - & - & Yes & Yes \\
\hline Applicable totally & No & No & No & No & No & No & No & No & No & No & Yes & Yes \\
\hline
\end{tabular}

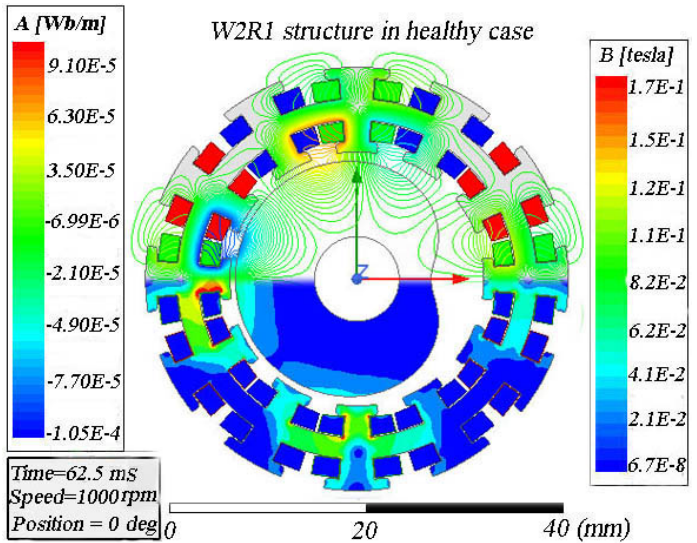

Fig. 9. Flux lines and flux density at a sample moment for healthy W2R1
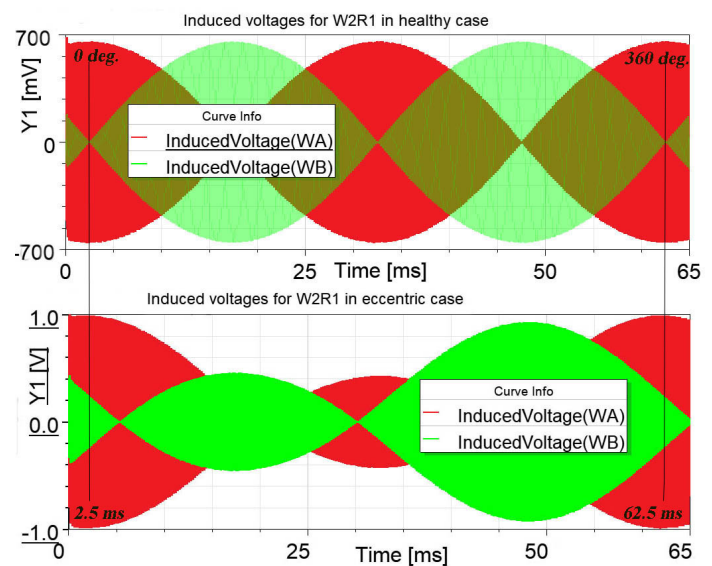

Fig. 10. Induced voltages for healthy and eccentric W2R1 under 1000 rpm obtained in Maxwell software

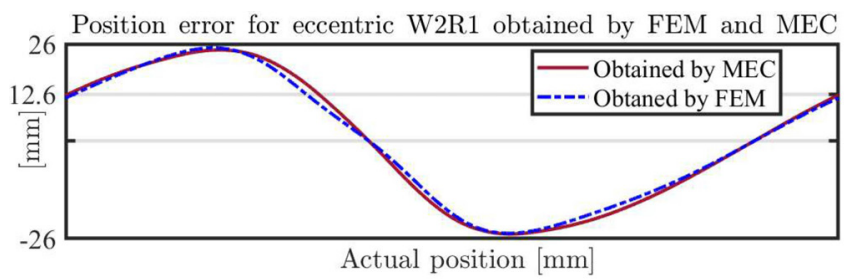

Fig. 11. Obtained position error for eccentric W2R1 by FEM and MEC

\section{EXPERIMENTAL RESULTS}

The analyzed resolver with two rotor shapes is manufactured for validation by the experimental setup. The $1-\mathrm{X}$ and 5 -X rotors in both healthy and eccentric cases are manufactured, where $0.25 \mathrm{~mm}$ eccentricity $\left(\sigma_{s}=0.5\right)$ is produced by the rotor center displacement in the manufacturing procedure as shown in Fig. 12. The resolvers tests are performed under $1000 \mathrm{rpm}$ rotor speed, where a $5 \mathrm{kHz} 4.2 V_{\mathrm{rms}}$, voltage is used by a signal generator as excitation. By using a $50 \mathrm{~Hz}$ universal motor, a 16000 -pulse optical encoder $\left(0.0225^{\circ}\right.$ resolution $)$ is used as the position reference. The induced voltages sampling is performed by a $200 \mathrm{kS} / \mathrm{s}$ Advantech Multifunction USB Module to data import, where the Hilbert transformation and a written $\mathrm{m}$-file are used for the envelopes computation.

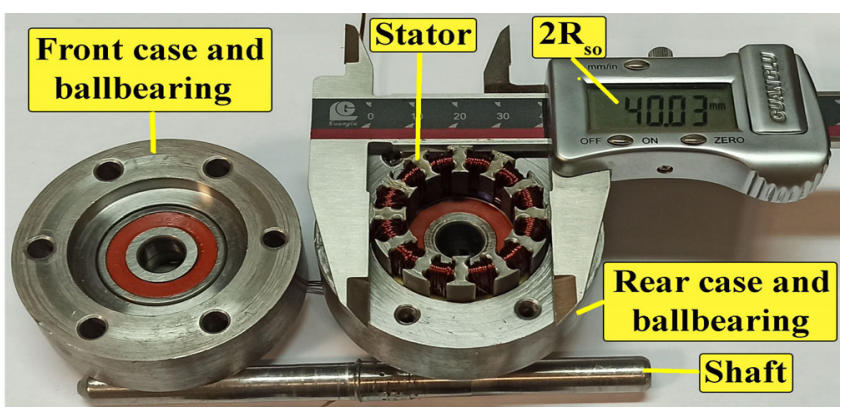

(a)

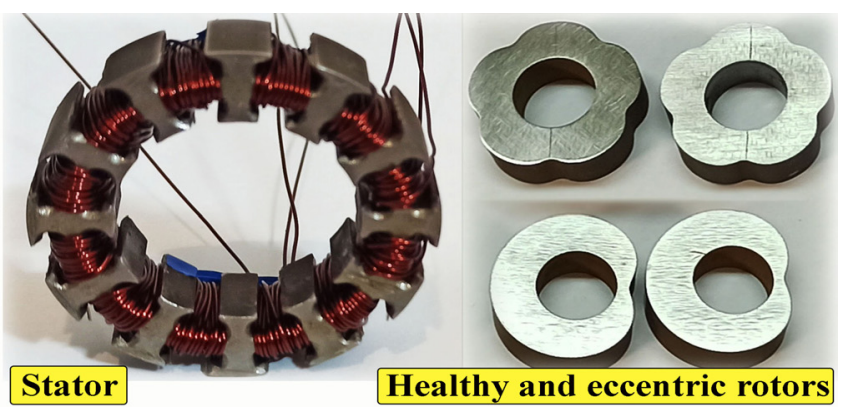

(b)

Fig. 12. Manufactured resolvers (a)-shaft, cases, ballbearings, and placed stator (b)- stator and rotors

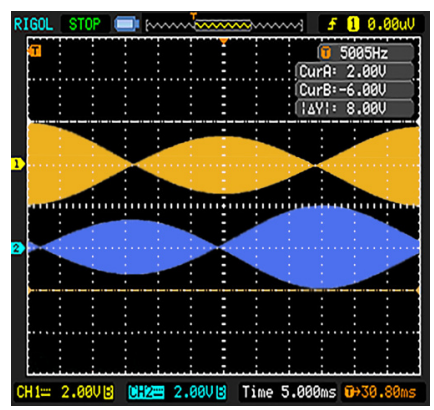

(a)

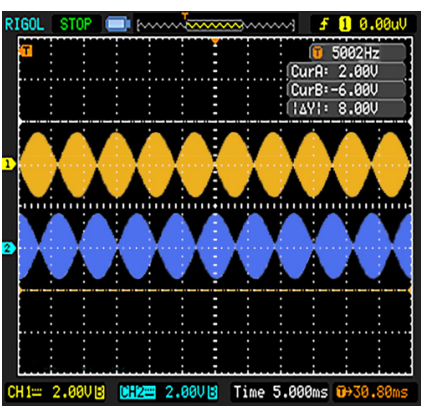

(b)
Fig. 13. Obtained $V_{\mathrm{Wsin}}$ and $V_{\mathrm{Wcos}}$ by experimental test with healthy and eccentric rotors (a)-for faulty W1R1 (b)-for healthy W3R5 
$\theta_{\text {error }}(t)$ for healthy W1R1 $\quad \theta_{\text {error }}(t)$ for eccentric W1R 1
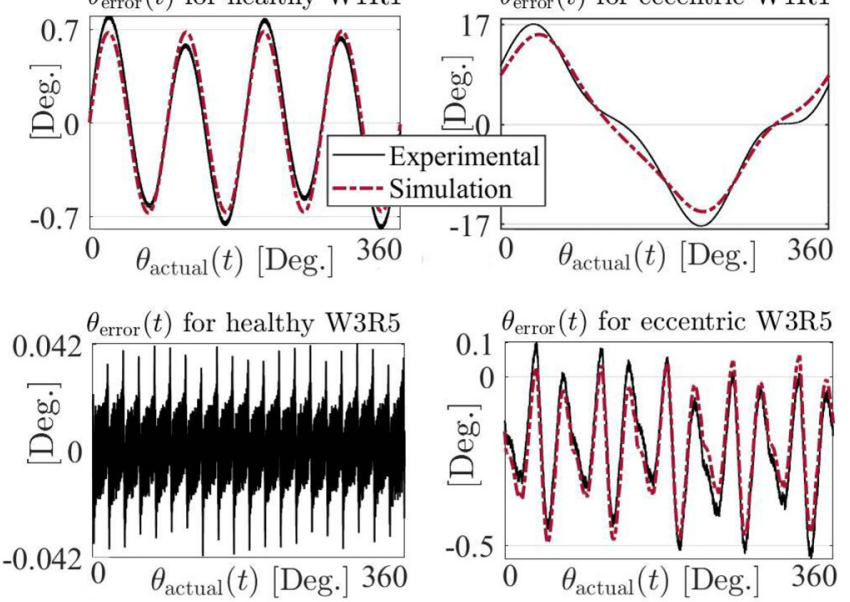

Fig. 14. Difference between the obtained position by the manufactured resolvers and optical encoder in healthy and eccentric cases

The induced voltages for the eccentric W1R1 and healthy W3R5 resolvers are shown in Figs. 13a and 13b. Moreover, the obtained position errors (difference between the obtained values by resolvers and encoder) are presented in Fig. 14. As can be seen in the figures, there is very good agreement between the obtained experimental and simulation results (see Figs. 3-7).

\section{CONCLUSION}

Variable reluctance resolver with toroidal windings is analyzed in this paper. Three windings types and four rotor shapes are considered for evaluation, where position error is analyzed in both healthy and faulty cases with static eccentricity fault. Regarding the analysis of the various structures, a well-known MEC method with adjustable accuracy is used for analysis thanks to its flexibility. Noticing the presented results, there are just two trustable structures that have high voltage peaks and low sensitivity to the eccentricity fault. Hence, a trustable resolver as an accurate position sensor can be obtained by sinusoidal windings configuration with $5-\mathrm{X}$ and $7-\mathrm{X}$ structures, where its sensitivity to the manufacturing tolerance is low. It is clarified that although the other configurations may have low position error in the healthy case, they are not practical due to high sensitivity to the eccentricity fault.

\section{REFERENCES}

[1] R. Alipour-Sarabi, Z. Nasiri-Gheidari, F. Tootoonchian, and H. Oraee, "Performance analysis of concentrated wound-rotor resolver for its applications in high pole number permanent magnet motors," IEEE Sensors Journal, vol. 17, no. 23, pp. 7877-7885, 2017.

[2] L. Xiao, Z. Li, and C. Bi, "An optimization approach to variable reluctance resolver,' IEEE Transactions on Magnetics, vol. 56, no. 2, pp. 1-5, 2020.

[3] Z. Wu and Y. Li, "High-accuracy automatic calibration of resolver signals via two-step gradient estimators," IEEE Sensors Journal, vol. 18, no. 7, pp. 2883-2891, 2018.

[4] M. Mohammad-Yari, M. Safari, R. Alipour-Sarabi, Z. Nasiri-Gheidari, and F. Tootoonchian, "Optimal winding selection for wound-rotor resolvers," Scientia Iranica, DOI 10.24200/sci.2019.52439.2764, pp. -, 2019. [Online]. Available: http://scientiairanica.sharif.edu/article_21510. html
[5] F. Abolqasemi-Kharanaq, R. Alipour-sarabi, Z. Nasiri-Gheidari, and F. Tootoonchian, "Magnetic equivalent circuit model for wound rotor resolver without rotary transformer's core," IEEE Sensors Journal, vol. 18, DOI 10.1109/JSEN.2018.2868682, no. 21, pp. 8693-8700, 2018.

[6] M. Alemi-Rostami, R. Alipour-Sarabi, G. Rezazadeh, Z. NasiriGheidari, and H. Oraee, "Design optimization of a double-stage resolver," IEEE Transactions on Vehicular Technology, vol. 68, DOI 10.1109/TVT.2019.2909096, no. 6, pp. 5407-5415, 2019

[7] S. Hajmohammadi, R. Alipour-Sarabi, Z. Nasiri-Gheidari, and F. Tootoonchian, "Influence of different installation configurations on the position error of a multiturn wound-rotor resolver," IEEE Sensors Journal, vol. 20, DOI 10.1109/JSEN.2020.2973225, no. 11, pp. 57855792, 2020.

[8] F. Tootoonchian, "Proposal of a new affordable 2-pole resolver and comparing its performance with conventional wound-rotor and vr resolvers," IEEE Sensors Journal, vol. 18, DOI 10.1109/JSEN.2018.2834552, no. 13 , pp. 5284-5290, 2018.

[9] H. Saneie, Z. Nasiri-Gheidari, and F. Tootoonchian, "Design and prototyping of a multi-turn sinusoidal air-gap length resolver," IEEE Transactions on Energy Conversion, vol. 35, DOI 10.1109/TEC.2019.2945120, no. 1 , pp. 271-278, 2020 .

[10] L. Xiao, Z. Li, and C. Bi, "An optimization approach to variable reluctance resolver," IEEE Transactions on Magnetics, vol. 56, DOI 10.1109/TMAG.2019.2953255, no. 2, pp. 1-5, 2020.

[11] F. Tootoonchian, "Design and optimization of a multi-turn variable reluctance resolver,' IEEE Sensors Journal, vol. 19, DOI 10.1109/JSEN.2019.2916915, no. 17, pp. 7275-7282, 2019.

[12] Q. Li, W. Sun, L. Sun, J. Yu, D. Xu, X. Jiang, and W. Geng, "Investigation of novel doubly salient pm variable reluctance resolvers," IEEE Access, vol. 7, pp. 104921-104 932, 2019.

[13] L. Sun, J. Taylor, A. D. Callegaro, and A. Emadi, "Stator-pmbased variable reluctance resolver with advantage of motional backemf," IEEE Transactions on Industrial Electronics, vol. 67, DOI 10.1109/TIE.2019.2955410, no. 11, pp. 9790-9801, 2020.

[14] M. Bahari, A. Davoodi, H. Saneie, F. Tootoonchian, and Z. NasiriGheidari, "A new variable reluctance pm-resolver," IEEE Sensors Journal, vol. 20, no. 1, pp. 135-142, 2019.

[15] H. Saneie, Z. Nasiri-Gheidari, and F. Tootoonchian, "Design-oriented modelling of axial-flux variable-reluctance resolver based on magnetic equivalent circuits and schwarz-christoffel mapping," IEEE Transactions on Industrial Electronics, vol. 65, no. 5, pp. 4322-4330, 2017.

[16] M. Bahari, Z. Nasiri-Gheidari, and F. Tootoonchian, "Modeling, performance analyzing, and prototyping of variable reluctance resolver with toroidal winding," IEEE Sensors Journal, vol. 21, DOI 10.1109/JSEN.2020.3030998, no. 4, pp. 4425-4432, 2021.

[17] H. Saneie, Z. Nasiri-Gheidari, F. Tootoonchian, and A. Daniar, "Simplified winding arrangement for integrated multi-turn resolvers," IEEE Transactions on Industrial Electronics, DOI 10.1109/TIE.2020.3039232, pp. 1-1, 2020.

[18] H. Saneie, R. Alipour-Sarabi, Z. Nasiri-Gheidari, and F. Tootoonchian, "Challenges of finite element analysis of resolvers," IEEE Transactions on Energy Conversion, vol. 34, DOI 10.1109/TEC.2018.2881465, no. 2, pp. 973-983, 2019.

[19] R. Alipour-Sarabi, Z. Nasiri-Gheidari, and H. Oraee, "Development of a 3-d magnetic equivalent circuit model for axial flux machines," IEEE Transactions on Industrial Electronics, 2019.

[20] R. Ghandehari, P. Naderi, and L. Vandevelde, "Performance analysis of a new type pm-resolver in healthy and eccentric cases by an improved parametric mec method," IEEE Transactions on Instrumentation and Measurement, vol. 70, DOI 10.1109/TIM.2021.3080388, pp. 1-10, 2021.

[21] P. Naderi, R. Ghandehari, and M. Heidary, "A comprehensive analysis on the healthy and faulty two types vr-resolvers with eccentricity and inter-turn faults," IEEE Transactions on Energy Conversion, DOI 10.1109/TEC.2021.3079725, pp. 1-1, 2021.

Peyman Naderi was born in Ahvaz, Iran, in 1975. He received his B.S. degree in Electronic Engineering in 1998 and M.S. degree in Power Engineering from Chamran University, Iran, Ahvaz in 2001. He has a Ph.D. degree in Power Engineering Science from K.N. Toosi University, Tehran, Iran. His interests are electrical machine modeling and fault diagnosis. He is currently associate professor in Shahid Rajaee Teacher Training University.

Reza Ghandehari was born in Isfahan, Iran, in 1971. He received M.Sc. and Ph.D. degrees in electrical engineering from Iran University of Science and Technology (IUST), Tehran, Iran, in 2002 and 2009, respectively. He is currently an assistant professor in the Electrical and Computer Engineering Department, Shahid Rajaee Teacher Training University, Iran. His current research interests include the electrical machines analysis and drive. 